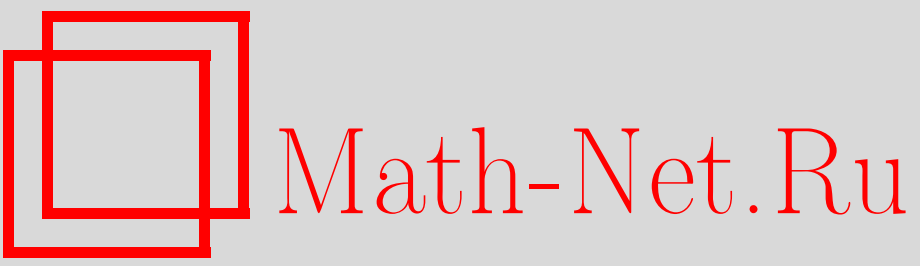

Ю. А. Горгинян, Л. А. Игнаточкина, Пространство аффинных связностей почти эрмитова многообразия, Итоги науки и техн. Сер. Соврем. мат. и ее прил. Tемат. обз., 2020, том 180, 31-40

DOI: https://doi.org/10.36535/0233-6723-2020-180-31-40

Использование Общероссийского математического портала Math-Net.Ru подразумевает, что вы прочитали и согласны с пользовательским соглашением

http://www.mathnet.ru/rus/agreement

Параметры загрузки:

IP : 54.84 .234 .179

26 апреля 2023 г., 02:30:12 


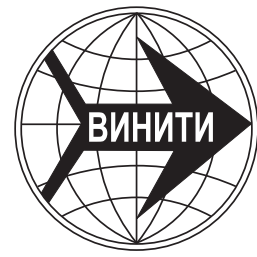

ИТОГИ НАУКИ И ТЕХНИКИ.

Современная математика и ее приложения.

Тематические обзоры.

Том 180 (2020). С. $31-40$

DOI: $10.36535 / 0233-6723-2020-180-31-40$

УДК 514.76

\title{
ПРОСТРАНСТВО АФФИННЫХ СВЯЗНОСТЕЙ ПОЧТИ ЭРМИТОВА МНОГООБРАЗИЯ
}

\author{
(c) 2020 г. Ю. А. ГОРГИНЯН, Л. А. ИГНАТОЧКИНА
}

\begin{abstract}
АннотАция. Рассмотрены аффинные связности, определяемые почти эрмитовой структурой гладкого многообразия. Доказано, что аффинное пространство рассмотренных связностей имеет размерность 12 тогда и только тогда, когда форма Ли почти эрмитовой структуры отлична от нуля. Найдены связности, которые задают постримановы геометрии. В классе $W_{4}$ найдены почти эрмитовы связности. Рассмотрено конформное преобразование почти эрмитовой структуры и аффинное отображение связностей, порождаемое этим преобразованием. Найдена связность, инвариантная относительно этого отображения.
\end{abstract}

Ключевые слова: аффинная связность, почти эрмитово многообразие, конформное преобразование.

\section{THE SPACE OF AFFINE CONNECTIONS OF AN ALMOST HERMITIAN MANIFOLD}

\author{
(c) 2020 YU. A. GORGINYAN, L. A. IGNATOCHKINA
}

\begin{abstract}
We consider affine connections determined by an almost Hermitian structure of a smooth manifold. We prove that the affine space of connections considered has dimension 12 if and only if the Lie form of the almost Hermitian structure is nonzero. We find connections that define post-Riemannian geometries and almost Hermitian connections in the class $W_{4}$. We examine a conformal transformation of an almost Hermitian structure and an affine mapping of connections generated by this transformation and find a connection invariant under this mapping.
\end{abstract}

Keywords and phrases: affine connection, almost Hermitian manifold, conformal transformation.

AMS Subject Classification: 53B05, 51E15

1. Введение. В настоящее время для решения задач в области космологии активно используется аппарат современной геометрии. Обобщенная теория гравитации потребовала использования многообразий, структура которых сложнее, чем структура пространств Римана (см. [1]). Пространство Римана характеризуется наличием псевдоримановой метрики и римановой связности. Риманова связность однозначно определяется метрикой: метрика в ней имеет нулевой ковариантный дифференциал и кручение этой связности равно нулю. Для проведения исследований в области космологии таких объектов на гладком многообразии оказалось недостаточно. Потребовалось ввести псевдориманову метрику и аффинную связность, не зависящую от нее. В этом случае кроме кривизны, появлялись еще две, вообще говоря, не нулевые характеристики связности: кручение и тензор неметричности. Пара, состоящая из псевдоримановой метрики и аффинной связности, называется аффинно-метрической структурой на многообразии. Среди аффинно-метрических структур выделяются те, для которых тензор неметричности распадается в тензорное произведение 1-формы и метрики. Добавляя к этому условию требования на тензор неметричности, либо на кручение, получают еще два вида аффинно-метрических структур, 
которые представляют интерес в физике. Этот интерес обусловлен тем, что в четырехмерных пространствах с аффинной связностью и метрикой лоренцевой сигнатуры причинная структура сохраняется тогда и только тогда, когда тензор неметричности распадается в тензорное произведение 1-формы и метрики (см. [1, с. 24]). Указанные аффинно-метрические структуры задают на многообразиях геометрии, которые называются постримановыми.

Классическим объектом в геометрии пространств Римана является конформное преобразование метрики. Оно однозначно определяет конформное преобразование римановой связности. В постримановых геометриях, так как метрика и связность независимы друг от друга, возникает необходимость в обобщении понятия конформного преобразования, а именно, кроме классического конформного преобразования метрики, требуется еще задание преобразования связности, зависящее или не зависящее от преобразования метрики. Основным критерием выбора таких преобразований в физике является сохранение причинной структуры. Различные виды обобщений конформных преобразований приведены в монографии [1].

В настоящей работе были рассмотрены четномерные многообразия с псевдоримановой метрикой и почти комплексной структурой, согласованной с ней. Такие многообразия называются почти эрмитовыми. Наличие почти комплексной структуры позволило построить некоторое конечномерное аффинное пространство аффинных связностей, в котором оказались связности, задающие постримановы геометрии для четномерных многообразий с псевдоримановой метрикой четной сигнатуры. Конформное преобразование почти эрмитовой структуры естественным образом определило множество отображений связностей, среди которых оказались как аналоги некоторых из приведенных в [1], так и не аналогичные им.

2. Почти эрмитовы многообразия и расслоение $A$-реперов. Почти эрмитовой струкmурой на гладком многообразии $M$ называется пара тензорных полей $(J, g)$, где $J$-тензорное поле типа $(1,1), J^{2}=-\mathrm{id}, g$ - псевдориманова метрика на $M$, согласованная с $J$, т.е. $g(J X, J Y)=g(X, Y)$ для любых векторных полей $X, Y$ на $M$ (см. [4]). Тензорное поле $J$ называется почти комплексной структурой.

Так как необходимым условиями существования почти эрмитовой структуры на гладком многообразии является его четномерность (а также ориентируемость), обозначим размерность $M$ через $2 n, n>1$.

На почти эрмитовом многообразии определяется кэлерова форма $F(X, Y)=g(J X, Y)$ и форма Ли $\alpha(X)=1 /(1-n) \delta F(J X)$, где $\delta$ - оператор кодифференцирования (см. [4,7]). Векторное поле $\xi$ двойственное форме Ли, т.е. $g(\xi, X)=\alpha(X)$, называется вектором Ли.

Договоримся, что индексы $a, b, c, d, \ldots$ (начало латинского алфавита) будут пробегать значения от 1 до $n$, индексы $i, j, k, l, \ldots$ (вторая половина латинского алфавита) будут пробегать значения от 1 до $2 n$ и $\hat{a}=a+n$.

Мы будем проводить исследования, используя присоединенную $G$-структуру, которая состоит из реперов $p=\left(m, \varepsilon_{1}, \ldots, \varepsilon_{n}, \varepsilon_{\hat{1}}, \ldots, \varepsilon_{\hat{n}}\right)$ или, короче, $p=\left(m, \varepsilon_{a}, \varepsilon_{\hat{a}}\right)$, где $a=1, \ldots, n$, $\hat{a}=n+1, \ldots, 2 n$. Они называются $A$-реперами. Векторы $A$-реперов получаются из ортонормированного относительно эрмитовой формы $\langle\langle X, Y\rangle\rangle=g(X, Y)+i g(X, J Y), X, Y \in T_{m}(M)$ репера $\left(m, e_{1}, \ldots, e_{n}\right)$ комплексного векторного пространства $T_{m}(M)$ по формулам

$$
\varepsilon_{a}=\sqrt{2} \sigma\left(e_{a}\right), \quad \varepsilon_{\hat{a}}=\sqrt{2} \bar{\sigma}\left(e_{a}\right),
$$

где

(см. [4]).

$$
\sigma=\frac{1}{2}(\mathrm{id}-i J), \quad \bar{\sigma}=\frac{1}{2}(\mathrm{id}+i J)
$$

Задание тензорного поля на многообразии $M$ равносильно заданию системы функций на пространстве расслоения реперов. Эти функции называются компонентами тензорного поля на пространстве расслоения $A$-реперов. В частности, для почти комплексной структуры $J$ система функций $\left\{J_{j}^{i}(p)\right\}$ на пространстве расслоения всех комплексных реперов определяется формулой $J_{m}^{\mathbb{C}}\left(\varepsilon_{i}\right)=J_{i}^{j}(p) \varepsilon_{j}$, а для псевдоримановой метрики $g$ система функций $\left\{g_{i j}(p)\right\}$ определяется формулой $g_{i j}(p)=g_{m}^{\mathbb{C}}\left(\varepsilon_{i}, \varepsilon_{j}\right)$. Здесь $p=\left(m, \varepsilon_{i}\right), m \in M$ - произвольный комплексный репер, знак $\mathbb{C}$ 
обозначает комплексификацию тензора, а $J_{m}^{\mathbb{C}}$ - значение тензорного поля $J^{\mathbb{C}}$ в точке $m$ (для $g$ аналогично).

На пространстве расслоения $A$-реперов эти системы функций имеют простой вид:

$$
\left(J_{j}^{i}\right)=\left(\begin{array}{cc}
i I_{n} & 0 \\
0 & -i I_{n}
\end{array}\right) \quad\left(g_{i j}\right)=\left(\begin{array}{cc}
0 & I_{n, s} \\
I_{n, s} & 0
\end{array}\right)
$$

где $I_{n, s}$ - матрица порядка $n$, на диагонали стоят числа 1 в количестве $n-s$ штук и -1 в количестве $s$ штук, $2 s$-индекс метрики $g$, а $i=\sqrt{-1}$ (см. [4]).

Обозначим через $\left\{\alpha_{a}, \alpha_{\hat{a}}\right\}$ компоненты формы Ли на пространстве расслоения $A$-реперов, а через $\left\{\xi^{b}, \xi^{\hat{b}}\right\}$ - компоненты вектора Ли $\xi$ на пространстве расслоения $A$-реперов. В силу вида компонент метрики $\left(g_{i j}\right)$ (см. (1)) очевидно, что

$$
\xi^{a}=\varepsilon(a) \alpha_{\hat{a}} ; \quad \xi^{\hat{a}}=\varepsilon(a) \alpha_{a},
$$

где $\varepsilon(a)=g_{a \hat{a}}$.

Для почти эрмитова многообразия определяются тензорные поля типа $(2,1)$ по формулам

$$
B(X, Y)=\frac{1}{4}\left(\nabla_{Y}(J)(J X)-\nabla_{J Y}(J) X\right), \quad C(X, Y)=-\frac{1}{4}\left(\nabla_{J Y}(J) X+\nabla_{Y}(J)(J X)\right),
$$

где $\nabla$ - риманова связность метрики $g$. Они называются виртуальным тензором и структурным тензором соответственно. Их ненулевые компоненты на пространстве расслоения $A$-реперов связаны с компонентами ковариантного дифференциала $J$ римановой связности по формулам

$$
\begin{aligned}
& B^{a b}{ }_{c}=-\frac{i}{2} \varepsilon(b) J_{\hat{b}, c}^{a} ; \quad B_{a b}{ }^{c}=\frac{i}{2} \varepsilon(b) J_{b, \hat{c}}^{\hat{a}} ; \quad \overline{B_{a b}{ }^{c}}=B^{a b}{ }_{c} . \\
& C_{a b c}=-\frac{i}{2} \varepsilon(b, c) J_{b, c}^{\hat{a}} ; \quad C^{a b c}=\frac{i}{2} \varepsilon(b, c) J_{\hat{b}, \hat{c}}^{a}, \quad \overline{C_{a b c}}=C^{a b c},
\end{aligned}
$$

где $\varepsilon(b, c)=g_{b \hat{b}} g_{c \hat{c}}$ (см. [4]). Компоненты вектора Ли и виртуального тензора связаны равенствами

$$
\xi^{a}=\frac{2}{n-1} B_{b}^{a b} ; \quad \xi^{\hat{a}}=\frac{2}{n-1} B_{a b}^{b}
$$

(см. [4]). Для классификации используется также альтернированный структурный тензор, который также называют структурным тензором. Его компоненты имеют вид

$$
B_{a b c}=C_{a[b c]} ; \quad B^{a b c}=C^{a[b c]} .
$$

Отметим, что в силу кососимметричности компонент $C_{a b c}$ по первым двум индексам, равенство $C_{a b c}=0$ равносильно равенству $B_{a b c}=0$.

3. Аффинно-метрическая геометрия гладкого многообразия. Пусть на гладком многообразии $M$ заданы псевдориманова метрика $g$, и аффинная связность $\nabla$. Тогда (см. [3, с. 312]) тензором неметричности называется тензорное поле $Q$, определяемое формулой

$$
Q(X, Y, Z)=-\nabla_{X}(g)(Y, Z)
$$

Говорят (см. [3, с. 313]), что на многообразии $M$ задана геометрия Римана-Картана-Вейля, если тензор неметричности имеет вид

$$
Q(X, Y, Z)=W(X) g(Y, Z)
$$

где $X, Y, Z$ - векторные поля на многообразии $M$. При этом 1-форма $W$ называется формой Вейля. Если тензор неметричности тождественно равен нулю, а кручение отлично от тождественного нуля, то говорят, что на многообразии задана геометрия Римана-Картана. Если тензор кручения равен нулю, а тензор неметричности имеет вид (6), то говорят, что на многообразии $M$ задана геометрия Римана-Вейля. Отметим, что используются также иные названия указанных геометрий (см., например, диаграмму [6, с. 170], [1, с. 6]). Многообразие с геометрией РиманаКартана-Вейля называется многообразием Вейля-Картана (Weyl-Cartan manifolds), а многообразия с геометрией Римана-Вейля названы многообразиями Вейля (Weyl manifolds). Указанные геометрии также называют постримановыми. 
4. Аффинное подпространство в пространстве аффинных связностей почти эрмитова многообразия. Пусть дано гладкое многообразие $M$ размерности $2 n, n>1$, и почти эрмитова структура $(J, g)$ на нем, $\alpha$ - форма Ли, $\xi$ - вектор Ли. Рассмотрим множество линейных связностей (короче, связностей)

$$
\begin{aligned}
\Delta_{X} Y=\nabla_{X} Y+\lambda_{1} \alpha(X) Y+\lambda_{2} \alpha(Y) X+ & \lambda_{3} \alpha(J X) Y+\lambda_{4} \alpha(J Y) X+ \\
+\lambda_{5} \alpha(X) J Y+\lambda_{6} \alpha(Y) J X+ & \lambda_{7} \alpha(J X) J Y+\lambda_{8} \alpha(J Y) J X+\lambda_{9} g(X, Y) \xi+ \\
& +\lambda_{10} g(J X, Y) \xi+\lambda_{11} g(X, Y) J \xi+\lambda_{12} g(J X, Y) J \xi,
\end{aligned}
$$

где $\lambda_{\beta}, \beta=1, \ldots, 12$ - произвольные вещественные числа.

Теорема 1. Если форма Ли а отлична от тождественного нуля, то множество связностей (7) является 12-мерным аффинным пространством А (12-мерной плоскостью в бесконечномерном афбинном пространстве связностей гладкого многообразия).

Доказательство. Рассмотрим линейную комбинацию

$$
\begin{aligned}
\lambda_{1} \alpha(X) Y+\lambda_{2} \alpha(Y) X+\lambda_{3} \alpha(J X) Y+\lambda_{4} \alpha(J Y) X+\lambda_{5} \alpha(X) J Y+ \\
+\lambda_{6} \alpha(Y) J X+\lambda_{7} \alpha(J X) J Y+\lambda_{8} \alpha(J Y) J X+\lambda_{9} g(X, Y) \xi+ \\
\quad+\lambda_{10} g(J X, Y) \xi+\lambda_{11} g(X, Y) J \xi+\lambda_{12} g(J X, Y) J \xi=0 .
\end{aligned}
$$

На пространстве расслоения $A$-реперов это равенство примет вид

$$
\begin{aligned}
\lambda_{1} \alpha_{i} \delta_{j}^{k}+\lambda_{2} \alpha_{j} \delta_{i}^{k}+\lambda_{3} \alpha_{l} J_{i}^{l} \delta_{j}^{k}+\lambda_{4} \alpha_{l} J_{j}^{l} \delta_{i}^{k}+ & \\
& +\lambda_{5} \alpha_{i} J_{j}^{k}+\lambda_{6} \alpha_{j} J_{i}^{k}+\lambda_{7} \alpha_{l} J_{i}^{l} J_{j}^{k}+\lambda_{8} \alpha_{l} J_{j}^{l} J_{i}^{k}+\lambda_{9} g_{i j} \xi^{k}+ \\
& +\lambda_{10} g_{l j} J_{i}^{l} \xi^{k}+\lambda_{11} g_{i j} J_{l}^{k} \xi^{l}+\lambda_{12} g_{l j} J_{i}^{l} J_{l}^{k} \xi^{l}=0 .
\end{aligned}
$$

В частности, при $i=a, j=b, k=c$ (индексы $i, j, k$ пробегают значения от 1 до $n$ ) с учетом (2) получим

$$
\left(\lambda_{1}-\lambda_{7}+i\left(\lambda_{3}+\lambda_{5}\right)\right) \alpha_{a} \delta_{b}^{c}+\left(\lambda_{2}-\lambda_{8}+i\left(\lambda_{4}+\lambda_{6}\right)\right) \alpha_{b} \delta_{a}^{c}=0 .
$$

Положим сначала $c=b$ и $c \neq a$, а затем $c=a$ и $c \neq b$. Тогда для почти эрмитовых многообразий, для которых форма Ли отлична от тождественного нуля, получаем

$$
\lambda_{1}-\lambda_{7}=0, \quad \lambda_{3}+\lambda_{5}=0, \quad \lambda_{2}-\lambda_{8}=0, \quad \lambda_{4}+\lambda_{6}=0 .
$$

Для случая $i=\hat{a}, j=b, k=c$ получаем

$$
\left(\lambda_{1}+\lambda_{7}-i \lambda_{3}+i \lambda_{5}\right) \varepsilon(a) \xi^{a} \delta_{b}^{c}+\left(\lambda_{9}+\lambda_{12}-i \lambda_{10}+i \lambda_{11}\right) \varepsilon(a) \xi^{c} \delta_{b}^{a}=0 .
$$

Аналогично предыдущему случаю, положив сначала $c=b$ и $c \neq a$, а затем $c=a$ и $c \neq b$, находим

$$
\lambda_{1}+\lambda_{7}=0, \quad \lambda_{5}-\lambda_{3}=0, \quad \lambda_{9}+\lambda_{12}=0, \quad \lambda_{10}-\lambda_{11}=0 ;
$$

Наконец, для случая $i=a, j=\hat{b}, k=c$ имеем

Тогда

$$
\left(\lambda_{2}+\lambda_{8}-i \lambda_{4}+i \lambda_{6}\right) \varepsilon(b) \xi^{b} \delta_{a}^{c}+\left(\lambda_{9}-\lambda_{12}+i \lambda_{10}+i \lambda_{11}\right) \varepsilon(b) \xi^{c} \delta_{a}^{b}=0 .
$$

$$
\lambda_{2}+\lambda_{8}=0, \quad \lambda_{6}-\lambda_{4}=0, \quad \lambda_{9}-\lambda_{12}=0, \quad \lambda_{11}+\lambda_{10}=0 .
$$

Объединяя уравнения (8), (9), (10), получим систему уравнений, из которой следует, что $\lambda_{\beta}=0$, $\beta=1, \ldots, 12$. Здесь мы учли, что форма Ли тождественно равна нулю тогда и только тогда, когда вектор Ли тождественно равен нулю, что и требовалось доказать.

Теорема 2. Для классов $\{0\}, W_{1}, W_{2}, W_{3}, W_{1} \oplus W_{2}, W_{1} \oplus W_{3}, W_{2} \oplus W_{3}, W_{1} \oplus W_{2} \oplus W_{3}$ почти эрмитовых многообразий из классификации Грея-Хервеллы (см. [4,7]) афффинное пространство аффинных связностей (7) тривиально (состоит только из римановой связности псевдоримановой метрики g). Для остальных восъми классов $W_{4}, W_{1} \oplus W_{4}, W_{2} \oplus W_{4}, W_{3} \oplus W_{4}, W_{1} \oplus W_{2} \oplus W_{4}$, $W_{1} \oplus W_{3} \oplus W_{4}, W_{2} \oplus W_{3} \oplus W_{4}$ (в случае, когда многообразие не содержится в менвшем классе из указанной классификации) пространство связностей (7) 12-мерно. 
В дальнейшем, если не оговорено противное, будем рассматривать нетривиальный случай.

Вычислим тензор неметричности (6) для связностей (7). Так как

$$
Q(X, Y, Z)=-\Delta_{X}(g)(Y, Z)=g\left(\Delta_{X} Y, Z\right)+g\left(Y, \Delta_{X} Z\right),
$$

то с учетом (7) получим

$$
\begin{array}{r}
Q(X, Y, Z)=2\left(\lambda_{1} \alpha(X)+\lambda_{3} \alpha(J X)\right) g(Y, Z)+\left(\lambda_{2}+\lambda_{9}\right)(\alpha(Y) g(X, Z)+\alpha(Z) g(X, Y))+ \\
+\left(\lambda_{4}-\lambda_{11}\right)(\alpha(J Y) g(X, Z)+\alpha(J Z) g(X, Y))+\left(\lambda_{6}+\lambda_{10}\right)(\alpha(Y) g(J X, Z)+\alpha(Z) g(J X, Y))+ \\
+\left(\lambda_{8}-\lambda_{12}\right)(\alpha(J Y) g(J X, Z)+\alpha(J Z) g(J X, Y)) .
\end{array}
$$

На пространстве расслоения $A$-реперов получим

$$
\begin{aligned}
Q_{i j k}=-g_{j k \mid i}= & 2\left(\lambda_{1} \alpha_{i}+\lambda_{3} \alpha_{l} J_{i}^{l}\right) g_{j k}+\left(\lambda_{2}+\lambda_{9}\right)\left(\alpha_{j} g_{i k}+\alpha_{k} g_{i j}\right)+ \\
& +\left(\lambda_{4}-\lambda_{11}\right)\left(\alpha_{l} J_{j}^{l} g_{i k}+\alpha_{l} J_{k}^{l} g_{i j}\right)+\left(\lambda_{6}+\lambda_{10}\right)\left(\alpha_{j} g_{l k} J_{i}^{l}+\alpha_{k} g_{l j} J_{i}^{l}\right)+ \\
& +\left(\lambda_{8}-\lambda_{12}\right)\left(\alpha_{l} J_{j}^{l} g_{t k} J_{i}^{t}+\alpha_{l} J_{k}^{l} g_{t j} J_{i}^{t}\right),
\end{aligned}
$$

где $\left\{g_{j k \mid i}\right\}$ - компоненты ковариантного дифференциала метрики $g$ в связности $\hat{\nabla}$. В частности, для $i=a, j=b, k=c$ получим

$$
Q_{a b c}=-g_{b c \mid a}=0
$$

При $i=\hat{a}, j=b, k=c$ получаем

$$
Q_{\hat{a} b c}=-g_{b c \mid \hat{a}}=\left(\lambda_{2}+\lambda_{9}+\lambda_{8}-\lambda_{12}+i\left(\lambda_{4}-\lambda_{11}-\lambda_{6}-\lambda_{10}\right)\right)\left(\alpha_{b} \delta_{c}^{a}+\alpha_{c} \delta_{b}^{a}\right) .
$$

При $i=a, j=\hat{b}, k=c$ получим

$$
Q_{a \hat{b} c}=-g_{\hat{b} c \mid a}=2\left(\lambda_{1}+i \lambda_{3}\right) \alpha_{a} \delta_{c}^{b}+\left(\lambda_{2}+\lambda_{9}-\lambda_{8}+\lambda_{12}+i\left(\lambda_{4}-\lambda_{11}+\lambda_{6}+\lambda_{10}\right)\right) \alpha_{c} \delta_{a}^{b} .
$$

Остальные компоненты тензора неметричности на пространстве расслоения $A$-реперов получаются из приведенных выше с использованием симметричности тензора неметричности по последним двум аргументам и комплексной сопряженности его компонент. Например, $Q_{\hat{a} b \hat{c}}=\bar{Q}_{a \hat{b} c}$.

Тензор кручения $S$ связности $\Delta$ из (7) имеет вид

$$
\begin{aligned}
S(X, Y)= & \left(\lambda_{1}-\lambda_{2}\right)(\alpha(X) Y-\alpha(Y) X)+\left(\lambda_{3}-\lambda_{4}\right)(\alpha(J X) Y-\alpha(J Y) X)+ \\
& +\left(\lambda_{5}-\lambda_{6}\right)(\alpha(X) J Y-\alpha(Y) J X)+\left(\lambda_{7}-\lambda_{8}\right)(\alpha(J X) J Y-\alpha(J Y) J X)+ \\
& +2 \lambda_{10} g(J X, Y) \xi+2 \lambda_{12} g(J X, Y) J \xi .
\end{aligned}
$$

На пространстве расслоения $A$-реперов получаем

$$
\begin{aligned}
S_{i j}^{k}=\left(\lambda_{1}-\lambda_{2}\right)( & \left.\alpha_{i} \delta_{j}^{k}-\alpha_{j} \delta_{i}^{k}\right)+\left(\lambda_{3}-\lambda_{4}\right)\left(\alpha_{l} J_{i}^{l} \delta_{j}^{k}-\alpha_{l} J_{j}^{l} \delta_{i}^{k}\right)+ \\
+\left(\lambda_{5}-\lambda_{6}\right)\left(\alpha_{i} J_{j}^{k}-\alpha_{j} J_{i}^{k}\right)+\left(\lambda_{7}-\lambda_{8}\right)\left(\alpha_{l} J_{i}^{l} J_{j}^{k}\right. & \left.-\alpha_{l} J_{j}^{l} J_{i}^{k}\right)+ \\
& +2 \lambda_{10} g_{l j} J_{i}^{l} \xi^{k}+2 \lambda_{12} g_{l j} J_{i}^{l} J_{t}^{k} \xi^{t} .
\end{aligned}
$$

Запишем существенные группы компонент тензора кручения (остальные группы компонент получаются ться из свойства кососимметричности тензора кручения и с помощью комплексного сопряжения):

$$
\begin{gathered}
S_{a b}^{c}=\left(\lambda_{1}-\lambda_{2}+\lambda_{8}-\lambda_{7}+i\left(\lambda_{3}-\lambda_{4}+\lambda_{5}-\lambda_{6}\right)\right)\left(\alpha_{a} \delta_{b}^{c}-\alpha_{b} \delta_{a}^{c}\right) ; \quad S_{a b}{ }^{\hat{c}}=0 . \\
S_{\hat{a} b}^{c}=\left(\lambda_{1}-\lambda_{2}+\lambda_{7}-\lambda_{8}+i\left(\lambda_{4}-\lambda_{3}+\lambda_{5}-\lambda_{6}\right)\right) \varepsilon(a) \xi^{a} \delta_{b}^{c}+2\left(\lambda_{12}-i \lambda_{10}\right) \xi^{c} \delta_{b}^{a} .
\end{gathered}
$$

Вычислим ковариантный дифференциал $J$ в связности $\Delta$. Так как

$$
\Delta_{X}(J) Y=\Delta_{X}(J Y)-J \Delta_{X} Y,
$$


с учетом (7) получим

$$
\begin{array}{r}
\Delta_{X}(J) Y=\nabla_{X}(J) Y+\left(\lambda_{2}+\lambda_{8}\right)(\alpha(J Y) X-\alpha(Y) J X)+\left(\lambda_{6}-\lambda_{4}\right)(\alpha(Y) X+\alpha(J Y) J X)+ \\
+\left(\lambda_{9}-\lambda_{12}\right)(g(X, J Y) \xi-g(X, Y) J \xi)+\left(\lambda_{10}+\lambda_{11}\right)(g(X, Y) \xi+g(X, J Y) J \xi) .
\end{array}
$$

На пространстве расслоения $A$-реперов получаем

$$
\begin{aligned}
J_{j \mid i}^{k}=J_{j, i}^{k}+\left(\lambda_{2}+\lambda_{8}\right)\left(\alpha_{l} J_{j}^{l} \delta_{i}^{k}\right. & \left.-\alpha_{j} J_{i}^{k}\right)+\left(\lambda_{6}-\lambda_{4}\right)\left(\alpha_{j} \delta_{i}^{k}+\alpha_{l} J_{j}^{l} J_{i}^{k}\right)+ \\
& +\left(\lambda_{9}-\lambda_{12}\right)\left(g_{i l} J_{j}^{l} \xi^{k}-g_{i j} J_{l}^{k} \xi^{l}\right)+\left(\lambda_{10}+\lambda_{11}\right)\left(g_{i j} \xi^{k}+g_{i l} J_{j}^{l} J_{m}^{k} \xi^{m}\right) .
\end{aligned}
$$

Запишем существенные группы компонент для ковариантного дифференциала $J$ в связности $\Delta$ (остальные группы компонент будут получаться с помощью комплексного сопряжения):

$$
\begin{gathered}
J_{b \mid a}^{c}=J_{b, a}^{c}=0 ; \quad J_{b \mid \hat{a}}^{c}=J_{b, \hat{a}}^{c}=0 ; \quad J_{b \mid a}^{\hat{c}}=J_{b, a}^{\hat{c}} ; \\
J_{\hat{b} \mid a}^{c}=J_{\hat{b}, a}^{c}+2\left(\lambda_{6}-\lambda_{4}+i\left(\lambda_{2}+\lambda_{8}\right)\right) \varepsilon(b) \xi^{b} \delta_{a}^{c}+2\left(\lambda_{10}+\lambda_{11}-i\left(\lambda_{9}-\lambda_{12}\right)\right) \varepsilon(b) \xi^{c} \delta_{a}^{b} .
\end{gathered}
$$

5. Структура пространства $A$-аффинных связностей. Пусть дано произвольное почти эрмитово многообразие, для которого пространство $A$ не тривиально. Выясним, есть ли среди связностей (7) те, которые задают вместе с метрикой $g$ геометрии Римана-Картана-Вейля, Римана-Картана, Римана-Вейля.

Тензор неметричности $Q$ имеет вид (6) тогда и только тогда, когда на пространстве расслоения $A$-реперов имеет вид $Q_{i j k}=W_{i} g_{j k}$. Существенными равенствами будут следующие (остальные будут получаться из них с помощью свойства симметричности $Q$ по последним индексам и с помощью комплексного сопряжения):

$$
Q_{a b c}=0 ; \quad Q_{\hat{a} b c}=0 ; \quad Q_{a \hat{b} c}=W_{a} \delta_{c}^{b} .
$$

Сравнивая эти равенства соответственно с (12), (13), (14), получаем, что тензор неметричности представим в виде (6) тогда и только тогда, когда

$$
\lambda_{2}+\lambda_{9}=0 ; \quad \lambda_{8}-\lambda_{12}=0 ; \quad \lambda_{4}-\lambda_{11}=0 ; \quad \lambda_{6}+\lambda_{10}=0 .
$$

Эти уравнения задают 8-мерную плоскость П в $A$ с координатами $\left(\lambda_{1}, \ldots, \lambda_{8}\right)$. Подставляя эти равенства в (11), получаем, что тензор неметричности $Q$ и форма Вейля $W$ в этом случае имеют вид

$$
Q(X, Y, Z)=2\left(\lambda_{1} \alpha(X)+\lambda_{3} \alpha(J X)\right) g(Y, Z) ; \quad W(X)=2\left(\lambda_{1} \alpha(X)+\lambda_{3} \alpha(J X)\right) .
$$

Рассмотрим $\Pi$ как декартово произведение 2-плоскости $\Sigma$ с координатами $\left(\lambda_{1}, \lambda_{3}\right) \equiv$ $\left(\lambda_{1}, 0, \lambda_{3}, 0, \ldots, 0\right)$ и 6 -плоскости $F$ с координатами $\left(\lambda_{2}, \lambda_{4}, \ldots, \lambda_{8}\right) \equiv\left(0, \lambda_{2}, 0, \lambda_{4}, \ldots, \lambda_{8}\right)$. Тогда П можно рассматривать как тотальное пространство тривиального расслоения с 2-мерной базой $\Sigma$ и с 6-мерными слоями -6-плоскостями, параллельными $F$. Проекция $\pi: \Pi \rightarrow \Sigma$ задается так: $\left(\lambda_{1}, \ldots, \lambda_{8}\right) \rightarrow\left(\lambda_{1}, \lambda_{3}\right)$. Каждый слой состоит из связностей, имеющих один и тот же тензор неметричности $Q$ и форму Вейля (21). Из формул $(21),(17),(16)$ следует, что слой, висящий над точкой $\lambda_{1}=0, \lambda_{3}=0$, состоит из связностей, задающих геометрию Римана-Картана и римановой связности.

Найдем связности в П, которые задают геометрию Римана-Вейля. Согласно (17), (16), (20) они задаются уравнениями

$$
\lambda_{1}-\lambda_{2}=0 ; \quad \lambda_{3}-\lambda_{4}=0 ; \quad \lambda_{5}=\lambda_{6}=\lambda_{7}=\lambda_{8}=0 .
$$

Это 2-плоскость, пересекающая каждый слой расслоения $(\Pi, \Sigma, F, \pi)$ в единственной точке. При этом базу $\Sigma$ (и висящий над ней слой) данная 2-плоскость пересекает только в римановой связности метрики $g$. Таким образом, 2-плоскость связностей, задающих геометрию РиманаВейля (и риманову геометрию), является образом некоторого сечения расслоения $(\Pi, \Sigma, F, \pi)$. Итак, доказана следующая теорема. 
Теорема 3. Множество связностей 12-мерного афбинного пространства $A$, задающих геометрию Римана-Картана-Вейля, является 8-плоскостью П. Эта 8-плоскость может быть рассмотрена как тривиальное расслоение с 2-мерной базой и 6-мерными слоями. Связности, задающие геометрию Римана-Картана (и риманова связность), составляют один из слоев этого расслоения. Множество связностей, задающих геометрию Римана-Вейля (и риманова связность), являются образом некоторого сечения этого расслоения.

6. Ковариантный дифференциал почти комплексной структуры. Напомним, что связность $\Delta$ является почти эрмитовой тогда и только тогда, когда $\Delta g=\Delta J=0$ (см. [5]). Отсюда следует, что почти эрмитовы связности определяют геометрию Римана-Картана. Из третьей группы равенств (18) вытекает, что, вообще говоря, в пространстве $A$ почти эрмитовых связностей нет.

Выясним, существуют ли классы из классификации Грея-Хервеллы почти эрмитовых структур, для которых в пространстве $A$ имеются почти эрмитовы связности.

Заметим, что в силу третьей группы равенств из (18) для выполнения равенства $\Delta J=0$ необходимо, чтобы $J_{a, b}^{\hat{c}}=0$. В силу (4) это равносильно $C_{c a b}=0$ или, эквивалентно, $B_{a b c}=0$. Кроме того, потребуем чтобы

$$
J_{\hat{b}, c}^{a}=2 i \varepsilon(b) B^{a b}{ }_{c}=2 i \varepsilon(b) \xi^{[a} \delta_{c}^{b]} .
$$

Эти условия определяют класс $W_{4}$ (см. [4]). Тогда из равенств (19) и (3) получаем, что для $\Delta J=0$ должно выполняться соотношение

$$
2 i \varepsilon(b) \xi^{[c} \delta_{a}^{b]}+2\left(\lambda_{6}-\lambda_{4}-i\left(\lambda_{2}+\lambda_{8}\right)\right) \varepsilon(b) \xi^{b} \delta_{a}^{c}+2\left(\lambda_{10}+\lambda_{11}-i\left(\lambda_{9}-\lambda_{12}\right)\right) \varepsilon(b) \xi^{c} \delta_{a}^{b}=0 .
$$

Тогда

$$
\left(i+2\left(\lambda_{10}+\lambda_{11}\right)-i\left(\lambda_{9}-\lambda_{12}\right)\right) \varepsilon(b) \xi^{c} \delta_{a}^{b}+\left(-i+2\left(\lambda_{6}-\lambda_{4}\right)-i\left(\lambda_{2}+\lambda_{8}\right)\right) \varepsilon(b) \xi^{b} \delta_{a}^{c}=0 .
$$

Рассуждая так же, как в теореме 1, получим

$$
\lambda_{10}+\lambda_{11}=0 ; \quad \lambda_{6}-\lambda_{4}=0 ; \quad \lambda_{9}-\lambda_{12}=\frac{1}{2} ; \quad \lambda_{2}+\lambda_{8}=-\frac{1}{2} .
$$

Для ковариантного постоянства метрики $g$ необходимо и достаточно выполнение условий (20) и $\lambda_{1}=\lambda_{3}=0$. Объединяя полученные уравнения и записывая только линейно независимые уравнения, получаем

$$
\lambda_{1}=\lambda_{3}=\lambda_{2}+\lambda_{9}=\lambda_{6}-\lambda_{4}=\lambda_{4}-\lambda_{11}=\lambda_{10}+\lambda_{11}=0 ; \quad \lambda_{2}+\lambda_{8}=-\frac{1}{2} ; \quad \lambda_{9}-\lambda_{12}=\frac{1}{2} .
$$

Эти уравнения задают 4-плоскость почти эрмитовых связностей. Итак, доказана следующая теорема.

Теорема 4. B пространстве $A$ для почти эрмитовых многообразий класса $W_{4}$ существует 4-плоскость почти эрмитовых связностей.

Теорема 5. В 8-плоскости П связностей, задающих геометрию Римана-Картана-Вейля, существует 6-плоскость связностей, для которых ковариантный дифберенииал почти комплексной структуры совпадает с ее ковариантным дифференииалом в римановой связности.

Доказательство. Действительно, из (19) следует, что $\Delta J=\nabla J$ тогда и только тогда, когда

$$
\left(\lambda_{6}-\lambda_{4}+i\left(\lambda_{2}+\lambda_{8}\right)\right) \varepsilon(b) \xi^{b} \delta_{a}^{c}+\left(\lambda_{10}+\lambda_{11}-i\left(\lambda_{9}-\lambda_{12}\right)\right) \varepsilon(b) \xi^{c} \delta_{a}^{b}=0 .
$$

Рассуждая аналогично доказательству теоремы 1 , получаем систему

$$
\lambda_{6}-\lambda_{4}=0 ; \quad \lambda_{2}+\lambda_{8}=0 ; \quad \lambda_{10}+\lambda_{11}=0 ; \quad \lambda_{9}-\lambda_{12}=0 .
$$

Объединяя ее с системой (20), получаем 6 линейно независимых уравнений

$$
\lambda_{4}=\lambda_{6} ; \quad \lambda_{11}=\lambda_{6} ; \quad \lambda_{10}=-\lambda_{6} ; \quad \lambda_{8}=\lambda_{9} ; \quad \lambda_{12}=\lambda_{9} ; \quad \lambda_{2}=-\lambda_{9} .
$$


Эти уравнения задают 6 -плоскость. Добавляя к этим уравнениям $\lambda_{1}=\lambda_{3}=0$, получаем 4-плоскость связностей, задающих геометрию Римана-Картана, причем ковариантный дифференциал $J$ в них совпадает с ковариантным дифференциалом $J$ в римановой связности.

7. Отображения пространства аффинных связностей. Пусть на многообразии $M$ задана почти эрмитова структура $(J, g)$. Рассмотрим конформно преобразованную почти эрмитову структуру $\left(J, \tilde{g}=e^{2 f} g\right)$, где $f$ - произвольная гладкая функция на многообразии $M$. Для нее построим множество связностей по формуле, аналогичной формуле (7):

$$
\begin{aligned}
\tilde{\Delta}_{X} Y=\tilde{\nabla}_{X} Y+\lambda_{1} \tilde{\alpha}(X) Y & +\lambda_{2} \tilde{\alpha}(Y) X+\lambda_{3} \tilde{\alpha}(J X) Y+ \\
+\lambda_{4} \tilde{\alpha}(J Y) X+ & \lambda_{5} \tilde{\alpha}(X) J Y+\lambda_{6} \tilde{\alpha}(Y) J X+\lambda_{7} \tilde{\alpha}(J X) J Y+\lambda_{8} \tilde{\alpha}(J Y) J X+ \\
& +\lambda_{9} \tilde{g}(X, Y) \xi+\lambda_{10} \tilde{g}(J X, Y) \tilde{\xi}+\lambda_{11} \tilde{g}(X, Y) J \tilde{\xi}+\lambda_{12} \tilde{g}(J X, Y) J \tilde{\xi}
\end{aligned}
$$

где $\lambda_{\beta}, \beta=1, \ldots, 12,-$ произвольные вещественные числа, $\tilde{\alpha}, \tilde{\xi}$ - форма Ли и вектор Ли преобразованной почти эрмитовой структуры, $\tilde{\nabla}$ - риманова связность псевдоримановой метрики $\tilde{g}$. Согласно теореме 1 множество таких связностей является 12-мерным аффинным пространством в случае $\tilde{\alpha} \neq 0$ и состоит из одной связности $\tilde{\nabla}$ в противном случае. Обозначим полученное множество связностей $\tilde{A}$.

Зададим отображение $\sigma$ пространства $A$ в пространство $\tilde{A}$, поставив в соответствие связности $\Delta$ из (7) связность $\tilde{\Delta}$ из (22) с теми же значениями $\lambda_{\beta}, \beta=1, \ldots, 12$. Найдем явное задание этого отображения.

При конформном преобразовании почти эрмитовой структуры форма Ли и вектор Ли преобразуются по формулам

$$
\tilde{\alpha}(X)=\alpha(X)+2 \beta(X) ; \quad \tilde{\xi}=e^{-2 f} \xi+2 e^{-2 f} \beta^{\sharp},
$$

где $\beta(X)=d f(X), \beta^{\sharp}$ - векторное поле, двойственное форме $\beta$, т.е. $g\left(X, \beta^{\sharp}\right)=\beta(X)$. Тогда из $(22)$ и (7) получим

$$
\begin{aligned}
\tilde{\Delta}_{X} Y=\Delta_{X} Y+\left(1+2 \lambda_{1}\right) \beta(X) Y+\left(1+2 \lambda_{2}\right) \beta(Y) X+\left(-1+2 \lambda_{9}\right) g(X, Y) \beta^{\sharp}+ \\
+2 \lambda_{3} \beta(J X) Y+2 \lambda_{4} \beta(J Y) X+2 \lambda_{5} \beta(X) J Y+2 \lambda_{6} \beta(Y) J X+2 \lambda_{7} \beta(J X) J Y+ \\
\quad+2 \lambda_{8} \beta(J Y) J X+2 \lambda_{10} g(J X, Y) \beta^{\sharp}+2 \lambda_{11} g(X, Y) J \beta^{\sharp}+2 \lambda_{12} g(J X, Y) J \beta^{\sharp} .
\end{aligned}
$$

Согласно теореме 2 пространство связностей $A$ не тривиально для классов вида $U \oplus W_{4}$, где $U=W_{1}, W_{2}$ и т. д., кроме $W_{4}$. В [7] для римановой метрики $g$ доказано, что для многообразий этих классов с замкнутой (соответственно, точной) формой Ли локально (соответственно, глобально) существует такое конформное преобразование метрики, что преобразованная почти эрмитова структура принадлежит классу $U$. В этих классах согласно той же теореме 2 пространство связностей $\tilde{A}$ будет состоять из единственной римановой связности метрики $\tilde{g}$. Например, для класса $\left(W_{1} \oplus W_{4}\right) \backslash W_{1}$ для каждого многообразия локально существует (и единственно) такое преобразование метрики $(J, \tilde{g})$, принадлежащее классу $W_{1}$ (см. [2]). В этом классе форма Ли тождественно равна нулю, и пространство связностей тривиально. Поэтому интерес представляет случай, когда аффинное пространство связностей $A$ исходного многообразия является 12 мерным, а пространство $\tilde{A}$ тривиально. Тогда из формул (23) и (24) получаем, что

$$
\tilde{\Delta}_{X} Y=\nabla_{X} Y-\frac{1}{2} \alpha(X) Y-\frac{1}{2} \alpha(Y) X+\frac{1}{2} g(X, Y) \xi .
$$

Согласно (11) тензор неметричности имеет вид

$$
Q(X, Y, Z)=-\alpha(X) g(Y, Z)
$$

а кручение равно нулю. Следовательно, эта связность задает геометрию Римана-Вейля.

Рассмотрим общий случай: обе формы Ли $\alpha$ и $\tilde{\alpha}$ отличны от нуля, $A$ и $\tilde{A}$ являются $12-$ мерными аффинными пространствами, метрики $g$ и $\tilde{g}$ являются псевдоримановыми. В этом случае отображение $\sigma$ будет биекцией. Кроме того, из определения этого отображения следует, что оно 
переведет 8-плоскость П связностей, задающих геометрию Римана-Картана-Вейля, в 8-плоскость $\tilde{\Pi}$, также задающих геометрию Римана-Картана-Вейля, базу $\Sigma-$ в базу $\tilde{\Sigma}$, сохраняя слои расслоения. Следовательно, является морфизмом расслоений.

Заметим, что если в формуле (24) положить

$$
\lambda_{3}=\lambda_{4}=\lambda_{5}=\lambda_{6}=\lambda_{7}=\lambda_{8}=\lambda_{10}=\lambda_{11}=\lambda_{12}=0, \quad-\lambda_{2}=\lambda_{9}=\frac{1}{2},
$$

то получится преобразование, которое рассматривалось в [8] в случае 4-мерного многообразия с лоренцевой метрикой и было названо расширенным конформным преобразованием.

Выясним, существуют ли инвариантные связности относительно отображения $\sigma$. Из $(24)$ следует, что связности $\Delta$ будет инвариантной тогда и только тогда, когда

$$
\begin{aligned}
&\left(1+2 \lambda_{1}\right) \beta(X) Y+\left(1+2 \lambda_{2}\right) \beta(Y) X+\left(-1+2 \lambda_{9}\right) g(X, Y) \beta^{\sharp}+2 \lambda_{3} \beta(J X) Y+ \\
&+2 \lambda_{4} \beta(J Y) X+2 \lambda_{5} \beta(X) J Y+2 \lambda_{6} \beta(Y) J X+2 \lambda_{7} \beta(J X) J Y+2 \lambda_{8} \beta(J Y) J X+ \\
&+2 \lambda_{10} g(J X, Y) \beta^{\sharp}+2 \lambda_{11} g(X, Y) J \beta^{\sharp}+2 \lambda_{12} g(J X, Y) J \beta^{\sharp}=0 .
\end{aligned}
$$

На пространстве расслоения $A$-реперов получим

$$
\begin{aligned}
&\left(1+2 \lambda_{1}\right) \beta_{i} \delta_{j}^{k}+\left(1+2 \lambda_{2}\right) \beta_{j} \delta_{i}^{k}+(-1+\left.2 \lambda_{9}\right) g_{i j}\left(\beta^{\sharp}\right)^{k}+2 \lambda_{3} \beta_{l} J_{i}^{l} \delta_{j}^{k}+ \\
&+2 \lambda_{4} \beta_{l} J_{j}^{l} \delta_{i}^{k}+2 \lambda_{5} \beta_{i} J_{j}^{k}+2 \lambda_{6} \beta_{j} J_{i}^{k}+2 \lambda_{7} \beta_{l} J_{i}^{l} J_{j}^{k}+2 \lambda_{8} \beta_{l} J_{j}^{l} J_{i}^{k}+ \\
&+2 \lambda_{10} g_{l j} J_{i}^{l}\left(\beta^{\sharp}\right)^{k}+2 \lambda_{11} g_{i j} J_{l}^{k}\left(\beta^{\sharp}\right)^{l}+2 \lambda_{12} g_{l j} J_{i}^{l} J_{t}^{k}\left(\beta^{\sharp}\right)^{t}=0 .
\end{aligned}
$$

Здесь четыре существенные группы равенств (остальные четыре группы получаются из них комплексным сопряжением). При $i=a, j=b, k=\hat{c}$ получим тривиальное тождество, поэтому выпишем только оставшиеся три группы:

$$
\begin{gathered}
\left(1+2 \lambda_{1}-2 \lambda_{7}+2 i \lambda_{3}+2 i \lambda_{5}\right) \beta_{a} \delta_{b}^{c}+\left(1+2 \lambda_{2}-2 \lambda_{8}+2 i \lambda_{4}+2 i \lambda_{6}\right) \beta_{b} \delta_{a}^{c}=0, \\
\left(1+2 \lambda_{1}+2 \lambda_{7}+2 i \lambda_{5}-2 i \lambda_{3}\right) \varepsilon(a)\left(\beta^{\sharp}\right)^{a} \delta_{b}^{c}+\left(-1+2 \lambda_{9}+2 \lambda_{12}+2 i \lambda_{11}-2 i \lambda_{10}\right) \varepsilon(a)\left(\beta^{\sharp}\right)^{c} \delta_{b}^{a}=0, \\
\left(1+2 \lambda_{2}+2 \lambda_{8}+2 i \lambda_{6}-2 i \lambda_{4}\right) \varepsilon(b)\left(\beta^{\sharp}\right)^{b} \delta_{a}^{c}+\left(-1+2 \lambda_{9}-2 \lambda_{12}+2 i \lambda_{10}+2 i \lambda_{11}\right) \varepsilon(b)\left(\beta^{\sharp}\right)^{c} \delta_{a}^{b}=0 .
\end{gathered}
$$

Пусть функция $f$, задающая конформное преобразование метрики $g$, не константа. Так как форма $\beta$ и двойственное векторное поле $\beta^{\sharp}$ отличны от нулевых, из последних трех равенств получаем систему уравнений

$$
\begin{aligned}
1+2 \lambda_{1}-2 \lambda_{7}=0 ; & \lambda_{3}+\lambda_{5}=0 ; & 1+2 \lambda_{2}-2 \lambda_{8}=0 ; & \lambda_{4}+\lambda_{6}=0 ; \\
1+2 \lambda_{1}+2 \lambda_{7}=0 ; & \lambda_{3}-\lambda_{5}=0 ; & -1+2 \lambda_{9}+2 \lambda_{12}=0 ; & \lambda_{11}-\lambda_{10}=0 ; \\
1+2 \lambda_{2}+2 \lambda_{8}=0 ; & \lambda_{6}-\lambda_{4}=0 ; & -1+2 \lambda_{9}-2 \lambda_{12}=0 ; & \lambda_{11}+\lambda_{10}=0 .
\end{aligned}
$$

Решая эту систему, получаем, что для отображения $\sigma$ существует единственная инвариантная связность $\Delta\left(\lambda_{1}=\lambda_{2}=-\lambda_{9}=-1 / 2\right.$, остальные $\lambda_{\beta}$ равны нулю):

$$
\Delta_{X} Y=\nabla_{X} Y-\frac{1}{2} \alpha(X) Y-\frac{1}{2} \alpha(Y) X+\frac{1}{2} g(X, Y) \xi .
$$

Из формулы (15) следует, что эта связность имеет нулевое кручение. Тензор неметричности имеет вид

$$
Q(X, Y, Z)=-\alpha(X) g(Y, Z) .
$$

Тем самым доказана следующая теорема.

Теорема 6. Пусть обе формы Ли исходного и конформно преобразованного почти эрмитова многообразия отличны от тождественного нуля. Тогда существует единственная связность (7), инвариантная относительно отображения $\sigma$. Она задает геометрию Римана-Вейля, и ее форма Вейля с точностъю до знака совпадает с формой Ли.

Заметим, что полученная связность совпадает со связностью, которая была получена в случае тривиального пространства $\tilde{A}$. 


\section{СПИСОК ЛИТЕРАТУРЫ}

1. Бабурова О. В., Фролов Б. Н. Математические основы современной теории гравитации. - М.: Прометей, 2012.

2. Игнаточкина Л. А. Локальное строение многообразий Вайсмана-Грея// Совр. мат. прилож. Геом. анал. - 2015. - 96. - С. 70-80.

3. Катанаев М. О. Геометрические методы в математической физике/ arXiv: $1311.0733 \mathrm{v} 3$ [math.ph] .

4. Кириченко В. Ф. Обобщенные классы Грея-Хервеллы и голоморфно-проективные преобразования обобщенных почти эрмитовых структур// Изв. РАН. Сер. мат. - 2005. - 69, № 5. - С. 107--132.

5. Кириченко В. Ф. Дифференциально-геометрические структуры на многообразиях. - Одесса: Печатный дом, 2013.

6. Степанов С. E., Гордеева И. А. Геометрия многообразий Римана-Картана// Вестн. КемГУ. - 2001. -3 , № 1. - C. 168-181.

7. Gray A., Hervella L. The sixteen classes of almost Hermitian manifolds and their linear invariants// Ann. Mat. Pura Appl. IV. - 1980. — 123. - P. 35-58.

8. Smalley L. L. Brans-Dicke-type models with nonmetricity// Phys. Rev. D. - 1986. - 33. - P. 3590-3593.

Горгинян Юлия Ашотовна

Московский педагогический государственный университет

E-mail: kiyulia0@gmail.com

Игнаточкина Лия Анатольевна

Московский педагогический государственный университет

E-mail: ignlia@gmail.com 